\title{
The new star forming site NGC 6334 IV (MM3)^
}

\author{
P. Persi ${ }^{1}$, M. Tapia ${ }^{2}$, M. Roth ${ }^{3}$, and M. Gómez ${ }^{4}$ \\ 1 INAF-IASF, Sez. di Roma, via Fosso del Cavaliere 100, 00133 Roma, Italia \\ e-mail: paolo.persi@iasf-roma.inaf.it \\ 2 Instituto de Astronomía, UNAM, Apartado Postal 877, Ensenada, Baja California, CP 22830, Mexico \\ e-mail: mt@astrosen.unam.mx \\ 3 Las Campanas Observatory, Carnegie Institution of Washington, Casilla 601, La Serena, Chile \\ e-mail: miguel@lco.cl \\ 4 Observatorio Astronómico de Córdoba, Laprida 854, 5000 Córdoba, Argentina \\ e-mail: mercedes@oac.uncor.edu
}

Received 1 September 2008 / Accepted 27 October 2008

\section{ABSTRACT}

\begin{abstract}
Aims. The eastern part of the massive star forming region NGC 6334 IV is characterized by the presence of the millimeter continuum sources MM3, MM4, and the optically thin HII region G351.24+0.65. Here we study the star formation in this part of the cloud. Methods. We imaged this region, here named NGC 6334 IV (MM3), at sub-arcsec resolution in $K \mathrm{~s}, \mathrm{H}_{2}, \mathrm{Br} \gamma$, and in the mid-infrared, from 8.9 to $12.7 \mu \mathrm{m}$. In addition, we used IRAC/Spitzer and ISOCAM images.

Results. From the analysis of the IRAC color-color and $H-K_{\mathrm{s}}$ versus $K_{\mathrm{s}}-$ [3.6] diagrams, we found 18 embedded very young stellar objects within an area of $75^{\prime \prime} \times 75^{\prime \prime}$. One of these sources, IR-MM3 (IRS 8E), shows a very steep energy distribution with an infrared spectral index of $\alpha_{\mathrm{IR}}=3.5$ and $L_{\mathrm{bol}}=985 L_{\odot}$. This protostar, which coincides with an $\mathrm{OH}$ maser source and with the continuum millimeter source MM3, is the exciting source of two hydrogen molecular knots found in our $\mathrm{H}_{2}$ image. Two sources (IRS 18, and 19) with infrared excess and a steep SED are probably associated with the HII region G351.24+0.65. The IRAC and ISOCAM images indicate the presence of extended PAH emission in the region.

Conclusions. We have discovered a new center of massive star formation in the giant molecular cloud complex NGC 6334.
\end{abstract}

Key words. stars: formation - ISM: HII regions - ISM: jets and outflows - ISM: clouds - infrared: stars - infrared: general

\section{Introduction}

NGC 6334 IV is one of the six known centres of massive star formation in the complex giant molecular cloud NGC 6334 (Persi \& Tapia 2008). It is characterized by the presence of a shell-like HII region found by Rodríguez et al. (1982) (radio source A) with two large radio bipolar plumes that extend approximately $2^{\prime}$ (Rodríguez et al. 1988). The same bipolar structure was observed in the near-infrared by Persi et al. (2000). At the center of this giant bipolar structure, two bright young stellar objects (HHS 19 and HHS 20) were found by Harvey et al. (1987). Both sources are extended and multiple as shown by high resolution near and mid-infrared images (Persi et al. 2000; Kraemer et al. 1999). In particular, IRS 20 is composed of at least two ZAMS B2-B3 stars.

(Sub)millimeter and millimeter continuum maps obtained by Sandell (1999) show this region to have a complex morphology, with the presence of at least four detected sources. MM1 is a double-peaked source approximately centered on the HII region $\mathrm{A}$, while MM2 roughly coincides with the protostar IRS 3, detected at $10 \mu \mathrm{m}$. Two other protostellar candidates (MM3 and MM4) are located in the eastern part of the complex. In particular, MM3 is a relatively compact source with a mass of $150 M_{\odot}$. Close to the millimeter peak MM3, Persi et al. (2000)

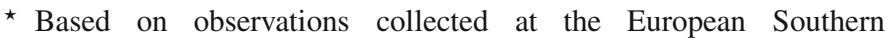
Observatory, La Silla, Chile, ESO proposal 073.C-0099(A), Las Campanas Observatory, Chile, and at the Observatorio Astronomico Nacional at San Pedro Mártir (Mexico).
}

found an infrared nebula with several point-like sources at $2.2 \mu \mathrm{m}$. This reflection nebula was named IRN IV-3 by Hashimoto et al. (2008), who obtained polarimetric maps in $J$, $H$ and $K_{\mathrm{s}}$. An $\mathrm{OH}$ maser source was found by Brooks \& Whiteoak (2001) at a distance of $\sim 3^{\prime \prime}$ from the MM3 peak. The faint optically thin HII region G351.24+0.65 found by Moran et al. (1990) lies roughly half way between MM3 and MM4.

All these observations suggest that this area of NGC 6334 IV is a new center of active star formation (Persi et al. 2000). In order to confirm this hypothesis and to characterize its young stellar objects, we present new higher resolution near and mid IR observations of the eastern part of NGC 6334 IV. IRAC/Spitzer and ISOCAM data will be used to supplement our ground based observations. These data are presented in Sect. 2, while in Sect. 3 we discuss the results. The conclusions are given in Sect. 4.

\section{Observations}

\subsection{Near-infrared images}

Near-infrared images at $K_{\mathrm{s}}, \operatorname{Br} \gamma(\lambda=2.165 \mu \mathrm{m} ; \Delta \lambda=$ $0.022 \mu \mathrm{m})$ and $\mathrm{H}_{2}(\lambda=2.125 \mu \mathrm{m} ; \Delta \lambda=0.024 \mu \mathrm{m})$, centered on RA $(\mathrm{J} 2000)=17^{\mathrm{h}} 20^{\mathrm{m}} 23.6^{\mathrm{s}}, \operatorname{Dec}(\mathrm{J} 2000)=-35^{\circ} 55^{\prime} 04.6^{\prime \prime}$, were collected on the night of 2003 May 15 using the Perssons Auxiliary Nasmyth Infrared Camera (PANIC) attached to the Magellan Clay $6.5 \mathrm{~m}$ telescope at Las Campanas Observatory (Chile). PANIC has a Rockwell $1024 \times 1024$ Hawaii array that provides a $2^{\prime} \times 2^{\prime}$ field of view with a $0.125^{\prime \prime} /$ pixel scale. We 


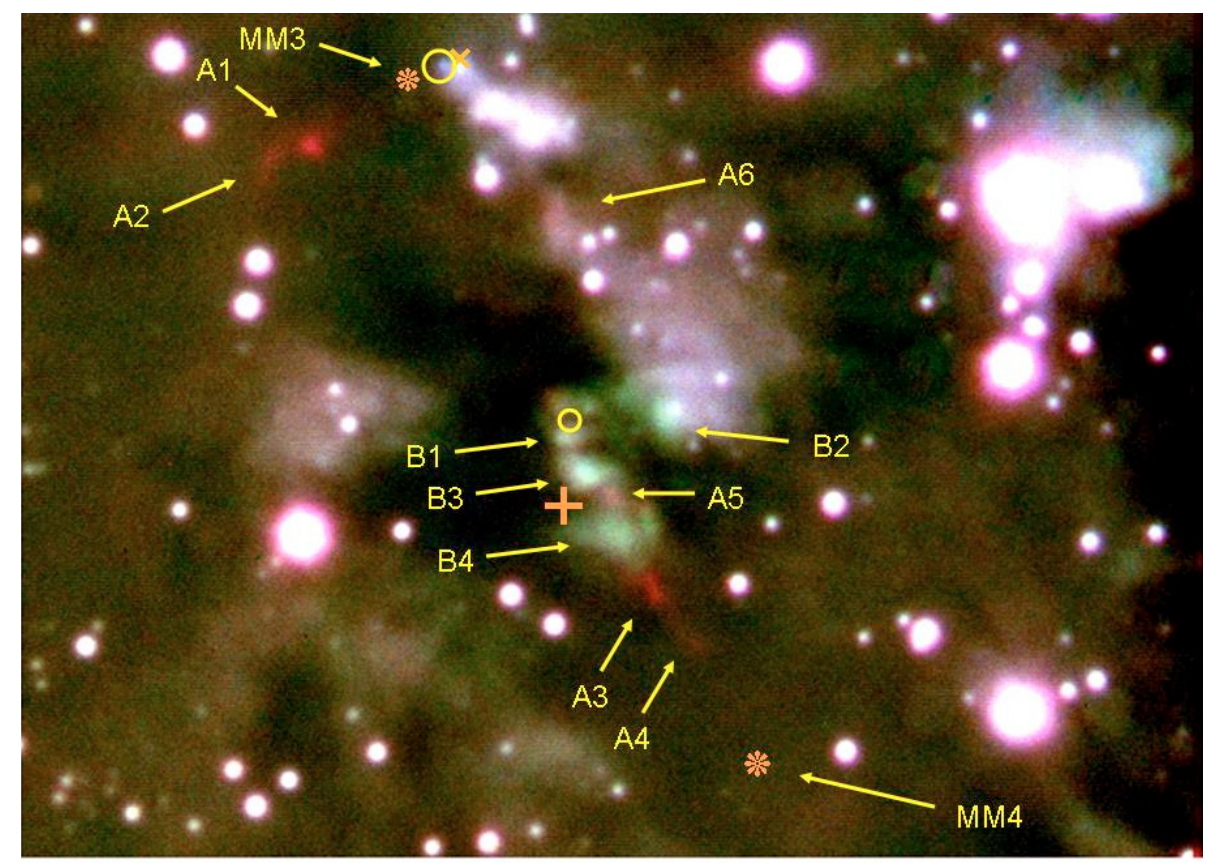

Fig. 1. 78" $\times 58^{\prime \prime}$ color-coded image of the region centered on RA(J2000) $=17^{\mathrm{h}} 20^{\mathrm{m}} 23.6^{\mathrm{s}}, \operatorname{Dec}(\mathrm{J} 2000)=-35^{\circ} 55^{\prime} 05^{\prime \prime}$ made from the $K_{\mathrm{s}}(\mathrm{blue})$, Br $\gamma$ (green), and $\mathrm{H}_{2}$ (red) individual images. North is the top and east to the left. The detected $\mathrm{H}_{2}$ knots are indicated with the letters A, while the letters B show the positions of the bright Br $\gamma$ emission knots. The millimeter sources MM3 and MM4 are marked with asterisks. The different symbols are: the HII region G351.24+0.65 (+), the OH maser (x), and the mid-IR sources detected from ground-based observations (o). The bluish extended emissions are reflection-dominated nebulae.

obtained nine dithered frames of $25 \mathrm{~s}$ in $K_{\mathrm{s}}$ and $120 \mathrm{~s}$ in $\mathrm{Br} \gamma$ and $\mathrm{H}_{2}$, offsetting the telescope by $6^{\prime \prime}$ between consecutive exposures. The measured PSF $(F W H M)$ was $\sim 0.7^{\prime \prime}$. The images were calibrated using standard stars from the list of Persson et al. (1998).

Figure 1 shows a color-coded image obtained by combining the $K_{\mathrm{s}}$ (blue), $\mathrm{Br} \gamma$ (green), and $\mathrm{H}_{2}$ (red) individual images of the region in MM3. From this figure, the presence of several infrared nebulosities in the region, including six hydrogen molecular knots (A1-A6 in Fig. 1) that will be discussed in the next sections, is evident. In addition, significant hydrogen recombination-line emission knots (B1-B4 in Fig. 1) are found around the radio HII region.

We have computed more accurate positions and new photometry of the red sources found by Persi et al. (2000) to be associated with the reflection nebula IRN IV-3. The aperture used was $1.1^{\prime \prime}$. With the present sub-arcsec resolution, we discovered a new source $1.3^{\prime \prime}$ east of IRS 8. This source, named IRS 8E, is within the positional uncertainty of the millimeter peak MM3. Fig. 2 illustrates the portion of the $K_{\mathrm{s}}$ image of this reflection nebula, while in Table 1 the coordinates and the $K_{\mathrm{s}}$ magnitudes of the red sources associated with IRN IV-3 are given.

\subsection{Ground-based mid-infrared images}

Mid-infrared images centered approximately at the same position as the near-infrared images were obtained on 2004 April 2 with TIMMI2 attached to the $3.6 \mathrm{~m}$ ESO telescope at La Silla (Chile). This thermal infrared camera uses a $320 \times 240$ pixel Si:As array manufactured by Raytheon. The images were taken at $8.9,9.8$ and $11.9 \mu \mathrm{m}$ with a scale of $0.2^{\prime \prime} /$ pix. The standard chop-nodding technique was used to remove the sky and telescope emission background. We employed a chop throw of $20^{\prime \prime}$, with a nodding offset of $20^{\prime \prime}$ perpendicular to the chop direction. The total on-source integration time was $24 \mathrm{~min}$ in all

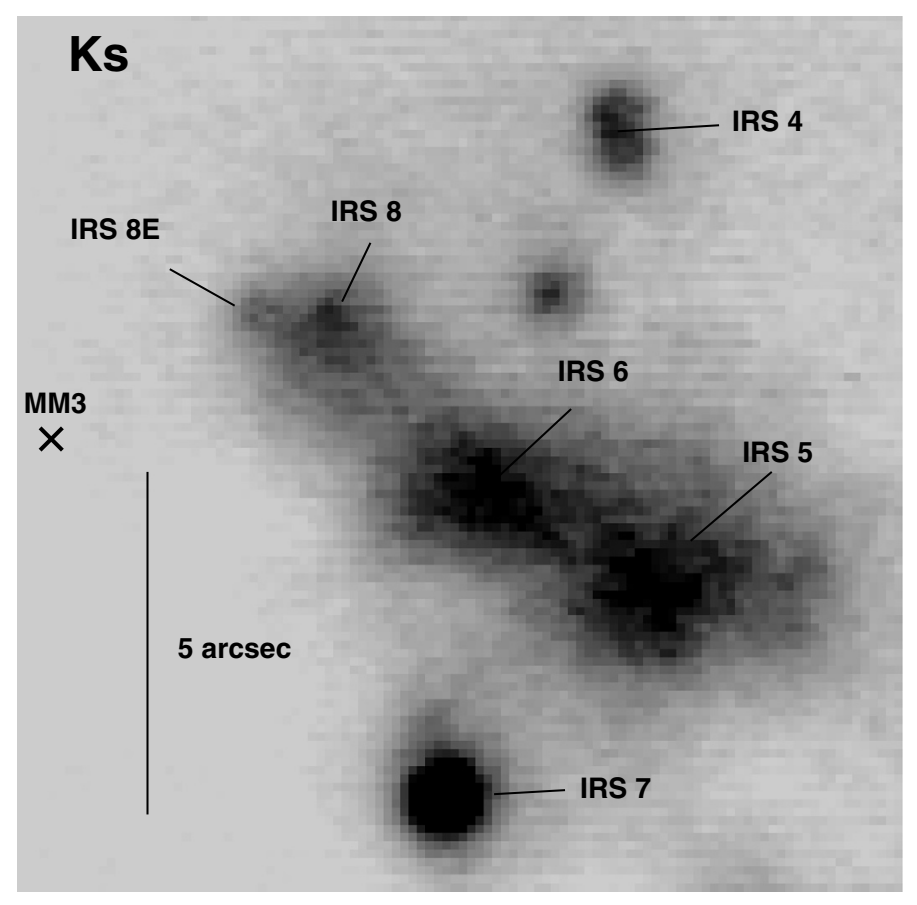

Fig. 2. A section of the Clay $K_{\mathrm{s}}$ image around the reflection nebula IRN IV 3. North is the top and east to the left.

filters. Standard stars listed in the TIMMI2 user's manual were observed at similar air masses for flux calibration. The measured PSFs $(F W H M)$ were $0.82^{\prime \prime}, 0.88^{\prime \prime}$, and $1.02^{\prime \prime}$ at, 8.9, 9.8, and $11.9 \mu \mathrm{m}$, respectively. The images at 8.9 and $11.9 \mu \mathrm{m}$, illustrated in Fig. 3, show the presence of two bright sources: IR-MM3, identified with the near-IR source IRS $8 \mathrm{E}$, and a diffuse emission source associated with the infrared reflection nebula IRN IV-6 (Hashimoto et al. 2008), which is clearly 

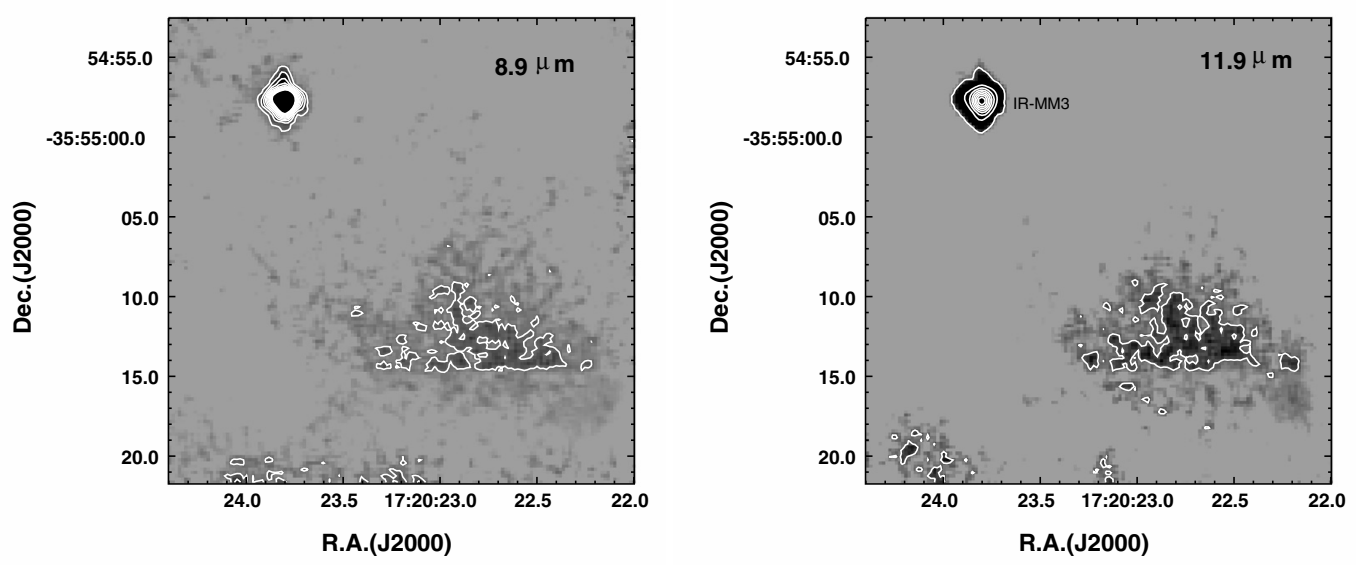

Fig. 3. TIMMI2 images at 8.9 and $11.9 \mu \mathrm{m}$.

Table 1. Coordinates and $K_{\mathrm{s}}$ magnitudes of the sources in the red nebula IRN IV-3.

\begin{tabular}{|c|c|c|c|c|}
\hline \multirow[t]{2}{*}{$\bar{~} \overline{\text { IRS }}$} & 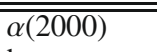 & 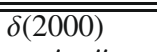 & \multirow{2}{*}{$\begin{array}{l}K_{\mathrm{s}} \\
\mathrm{mag}\end{array}$} & \multirow[t]{2}{*}{ Note } \\
\hline & $\mathrm{h} \quad \mathrm{m} \quad \mathrm{s}$ & & & \\
\hline 4 & 172023.40 & -355453.9 & 15.13 & Extended source \\
\hline 5 & 172023.39 & -355500.5 & 14.73 & Refl. Neb. (2.4") \\
\hline 6 & 172023.55 & -355459.4 & 14.85 & Refl. Neb. (1.3") \\
\hline 7 & 17202361 & -355403.9 & 14.38 & Point-like \\
\hline 8 & 172023.74 & -355456.9 & 14.83 & Refl. Neb (1.4") \\
\hline $8 \mathrm{E}$ & 172023.84 & -355456.7 & 15.25 & IR-MM3 \\
\hline
\end{tabular}

Table 2. Mid-infrared flux densities of the source IR-MM3 obtained with different instruments and apertures.

\begin{tabular}{lcll}
\hline \hline $\begin{array}{l}\text { Wavelengths } \\
(\mu \mathrm{m})\end{array}$ & $\begin{array}{c}\text { Flux } \\
(\mathrm{Jy})\end{array}$ & Instrument/Telescope & $\begin{array}{l}\text { Ap. } \\
\left({ }^{\prime \prime}\right)\end{array}$ \\
\hline 3.6 & $0.27(0.01)$ & IRAC/Spitzer & 3.7 \\
4.5 & $1.25(0.08)$ & IRAC/Spitzer & 3.7 \\
5.8 & $2.6(0.1)$ & ISOCAM & 9 \\
6.8 & $2.4(0.2)$ & ISOCAM & 9 \\
8.9 & $1.68(0.16)$ & TIMMI2/ESO 3.6 m & 2 \\
8.9 & $1.1(0.3)$ & CID/SPM 2.1 m & 5 \\
9.8 & $0.39(0.08)$ & TIMMI2/ESO 3.6 m & 2 \\
9.9 & $\leq 0.6$ & CID/SPM 2.1 m & 5 \\
$N(10.8)$ & $3.0(0.6)$ & CID/SPM 2.1 m & 5 \\
11.9 & $1.50(0.09)$ & TIMMI2/ESO 3.6 m & 2 \\
11.9 & $1.4(0.4)$ & CID/SPM 2.1 m & 5 \\
12.7 & $4.9(1.1)$ & CID/SPM 2.1 m & 5 \\
14.8 & $3.8(0.4)$ & ISOCAM & 9 \\
\hline
\end{tabular}

present in our near-infrared images of Fig. 1. A second point-like and very faint source, named MIRS 2, was detected at $\mathrm{RA}(\mathrm{J} 2000)=17^{\mathrm{h}} 20^{\mathrm{m}} 23.14^{\mathrm{s}}, \operatorname{Dec}(\mathrm{J} 2000)=-35^{\circ} 55^{\prime} 21.5^{\prime \prime} . \mathrm{We}$ obtained photometry of IR-MM3, MIRS 2 and of the extended source using the DAOPHOT package within IRAF (Stetson 1987). We used an aperture of $2^{\prime \prime}$ for the point-like sources and $6^{\prime \prime}$ for the extended source. The observed flux densities for the extended emission are $2.8 \mathrm{Jy}$ at $8.9 \mu \mathrm{m}$ and $3.1 \mathrm{Jy}$ at $11.9 \mu \mathrm{m}$, while for MIRS 2 , we measured a flux density at $11.9 \mu \mathrm{m}$ of $0.20 \pm 0.06 \mathrm{Jy}$. The results for IR-MM3 are given in Table 2.

Images between 8.9 and $12.7 \mu \mathrm{m}$ were also taken during the nights of 2000 May 29, 30 and 31 with the mid- infrared camera CID (Salas et al. 2006) on the $2.1 \mathrm{~m}$ telescope of the Observatorio Astronómico Nacional at San Pedro Mártir, Baja
California, Mexico. The camera is equipped with a Rockwell $128 \times 128$ pixel Si:As BIB detector array and delivers an effective scale of $0.55^{\prime \prime} /$ pix. The chop-nodding technique was used to remove the sky and telescope emission background. The standard stars $\alpha$ Boo, $\alpha$ Sco and $\beta$ Leo were observed for flux calibration. The measured point-spread function (PSF) ranged from about 1.5 to $1.8^{\prime \prime}(F W H M)$. The images are consistent with those obtained with TIMMI2. The photometry of the point-like source IR-MM3 was obtained in the all filters using an aperture of $5^{\prime \prime}$ and the results are reported in Table 2.

\subsection{IRAC/Spitzer images}

We retrieved images of the region NGC 6334 IV (MM3) at 3.6, 4.5, 5.8 and $8.0 \mu \mathrm{m}$ from the GLIMPSE survey archive (Benjamin et al. 2003) that imaged the inner Galactic plane with the IRAC camera (Fazio et al. 2004) on the Spitzer Space Telescope (Werner et al. 2004) with resolutions from 1.5" $(3.6 \mu \mathrm{m})$ to $1.9^{\prime \prime}(8.0 \mu \mathrm{m})$. Figure 4 (left panel) illustrates a composite "true-color" image $(3.6,4.5$, and $5.8 \mu \mathrm{m}$ in the blue, green and red channels, repectively) of NGC 6334 IV (MM3).

Diffuse emission, mainly between 5.8 and $8.0 \mu \mathrm{m}$ and probably due to the strong PAH features in these bands, dominates the IRAC and ISOCAM images (see also next section). Apart from IR-MM3 (Table 2), seventeen point-like sources were detected at $3.6 \mu \mathrm{m}$, of which $76 \%$ were detected in all four IRAC bands. We performed photometry of these sources using the DAOPHOT package within IRAF with an aperture of 3 pixels $\left(\sim 3.7^{\prime \prime}\right)$ and a sky annulus extending from 3 to 7 pixels. We applied the corresponding aperture corrections and the zero magnitude fluxes from the Spitzer/IRAC User Manual v.5.0. The source IR-MM3 is saturated in the 5.8 and $8.0 \mu \mathrm{m}$ images and its flux density at 3.6 and $4.5 \mu \mathrm{m}$ is reported in Table 2 .

\subsection{ISOCAM images}

Images centered at $\mathrm{RA}(\mathrm{J} 2000)=17^{\mathrm{h}} 20^{\mathrm{m}} 6.2^{\mathrm{s}}$, $\operatorname{Dec}(\mathrm{J} 2000)=-35^{\circ} 55^{\prime} 50^{\prime \prime}$ were collected through the 5.8, 6.8 , and $14.8 \mu \mathrm{m}$ narrow-band filters using the infrared camera ISOCAM (Cesarsky et al. 1996) aboard the Infrared Space Observatory (ISO) satellite (Kessler et al. 1996). The images were taken with a PFOV of $3^{\prime \prime}$ in CAM 01 observing mode. We obtained these images (TDT No. 64801721) from the ISO Data Archive Center. The ISOCAM "true-color" image made from the individual $5.8 \mu \mathrm{m}$ (blue), $6.8 \mu \mathrm{m}$ (green), and $14.8 \mu \mathrm{m}$ 

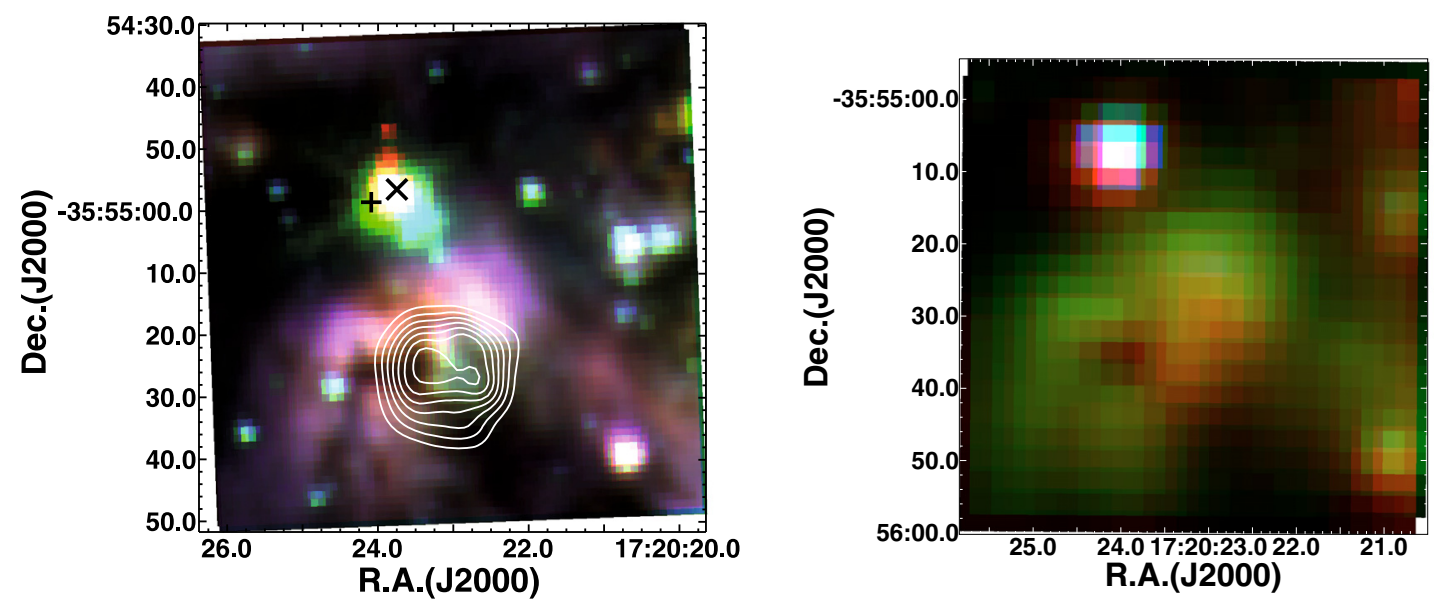

Fig. 4. Left panel: $75 " \times 75 "$ GLIMSPE/IRAC "true-color" image made from $3.6 \mu \mathrm{m}$ (blue), $4.5 \mu \mathrm{m}$ (green), and $5.8 \mu \mathrm{m}$ (red) images of NGC 6334 IV (MM3) centered as in Fig. 1. The contours represet the $3.6 \mathrm{~cm}$ radio source G351.24+0.65 (De Pree et al. 1995). The positions of the MM3 peak (+) (Sandell 1999) and of the OH maser (X) detected by Brooks \& Whiteoak (2001) are reported. Right panel: ISOCAM "true-color" image made from the $5.8 \mu \mathrm{m}$ (blue), $6.8 \mu \mathrm{m}$ (green), and $14.8 \mu \mathrm{m}$ (red) frames of the same region.

(red) ones is shown in Fig. 4 (right panel). The very bright nebulous emission in the 5.8 and $6.8 \mu \mathrm{m}$ bands confirms the presence of PAH emission. The photometry of the point-like source IR-MM3 (Table 2) was obtained with an aperture of $9^{\prime \prime}$.

\section{Discussion}

\subsection{Young stellar population}

The region east of NGC $6334 \mathrm{~A}$ is mainly characterized by the presence of two millimeter sources (MM3 and MM4) detected by Sandell (1999), the optically thin HII region G351.24+0.65 ionized by a B0 zero-age main-sequence (ZAMS) star (De Pree et al. 1995) and by an $\mathrm{OH}$ maser source (Brooks \& Whiteoak 2001) which coincides with our mid-IR source IR-MM3. Figure 5 shows these signposts of massive star formation on our Clay $K_{\mathrm{s}}$ image.

In order to study the young stellar population in this region, we analyzed the IRAC color-color diagram ([3.6]-[4.5] versus [5.8]-[8.0]) obtained for the point-like sources detected in the four colors (Fig. 6, left panel) and the $H-K_{\mathrm{s}}$ versus $K_{\mathrm{s}}-$ [3.6] (Fig. 6, right panel). We used the $H$ and $K_{\mathrm{s}}$ photometry reported by Persi et al. (2000) in order to construct this diagram.

Most of the sources have IRAC colors [5.8]-[8.0] $\geq 1.1$ and [3.6]-[4.5] $\leq 0.8$ and lie outside the locus of Class II YSOs, as indicated by Allen et al. (2004). IRAC sources with similar colors were found by Persi et al. (2006) in the high-mass star forming region $\mathrm{W} 75 \mathrm{~N}$. Jones et al. (2005) suggest that these sources represent high-mass versions of the Class I YSOs.

In the $H-K_{\mathrm{s}}$ versus $K_{\mathrm{s}}-$ [3.6] diagram, it is clear that a significant fraction of the sources lie to the right of the reddening vector (Fig. 6 right panel) indicating the presence of large infrared excesses. Combining the information of these two diagrams, we conclude that 18 sources in an area of $75^{\prime \prime} \times 75^{\prime \prime}$ containing the millimeter continuum sources MM3 and MM4 and the HII region G351.24+0.65 are young stellar objects. The young star IR-MM3 (IRS 8E) does not appear in Fig. 6 because it was detected only in $K_{\mathrm{S}}$ and it is saturated in the 5.8 and $8.0 \mu \mathrm{m}$ IRAC bands (see Fig. 4 left panel). The IRAC/Spitzer photometry and the coordinates of the sources with infrared excess together with their $K_{\mathrm{s}}$ magnitudes derived from our image are given in Table 3 . The $H$ magnitudes in the table are taken from Persi et al. (2000). To avoid confusion, the identification

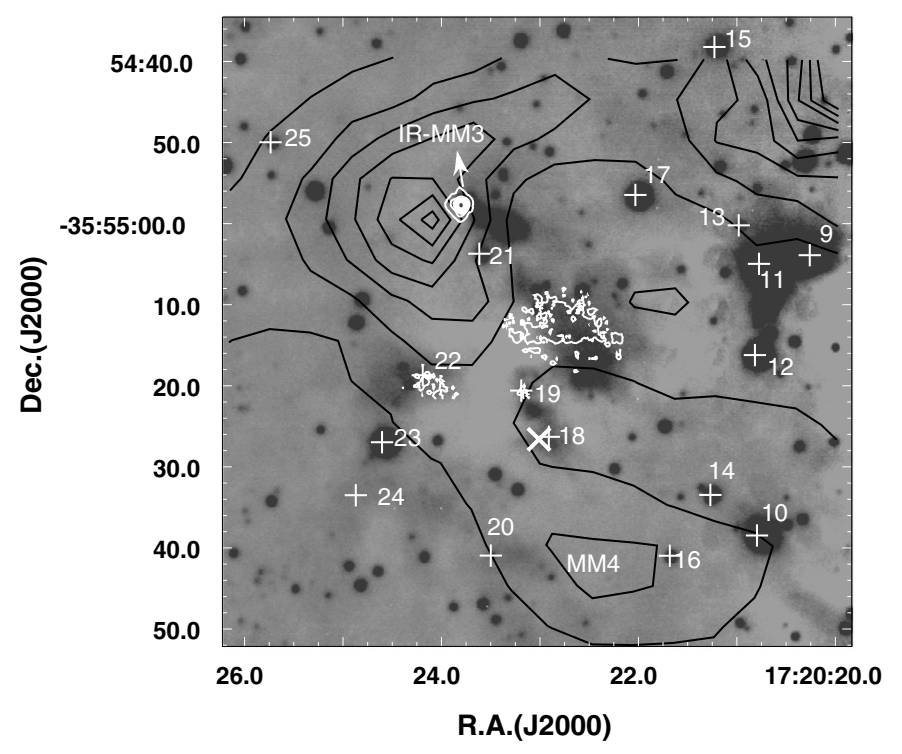

Fig. 5. $K_{\mathrm{s}}$ image taken with the $6.5 \mathrm{~m}$ Clay telescope with contours of $1.1 \mathrm{~mm}$ emission (black), and $11.9 \mu \mathrm{m}$ (white) overlaid. The sources with infrared excess are indicated with the symbol $(+)$ and numbered according to Table 3. The cross indicates the central position of the $3.6 \mathrm{~cm}$ radio continuum (De Pree et al. 1995).

numbering is a continuation of that initiated in Table 3 of Persi et al. (2000). Their positions are marked with crosses in Fig. 5 and identified with the numbers of Table 3 . Four sources with no infrared excess shown in Fig. 6 (right panel) are not reported in Table 3. This analysis confirms that NGC 6334 IV (MM3) is a very active site of star formation.

All the sources reported in Table 3 were detected in the near-infrared by Persi et al. (2000), and a few have been identified with the infrared sources (HHS) reported by Harvey et al. (1987). IRS 16 lies close to the millimeter source MM4 and shows a steep infrared energy distribution (Fig. 9), while IRS 18 and IRS 19 are within the boundaries of the HII region. In the next sub-sections we will discuss individually the properties of source IR-MM3 and of the those associated with the HII region G351.24+0.65. 

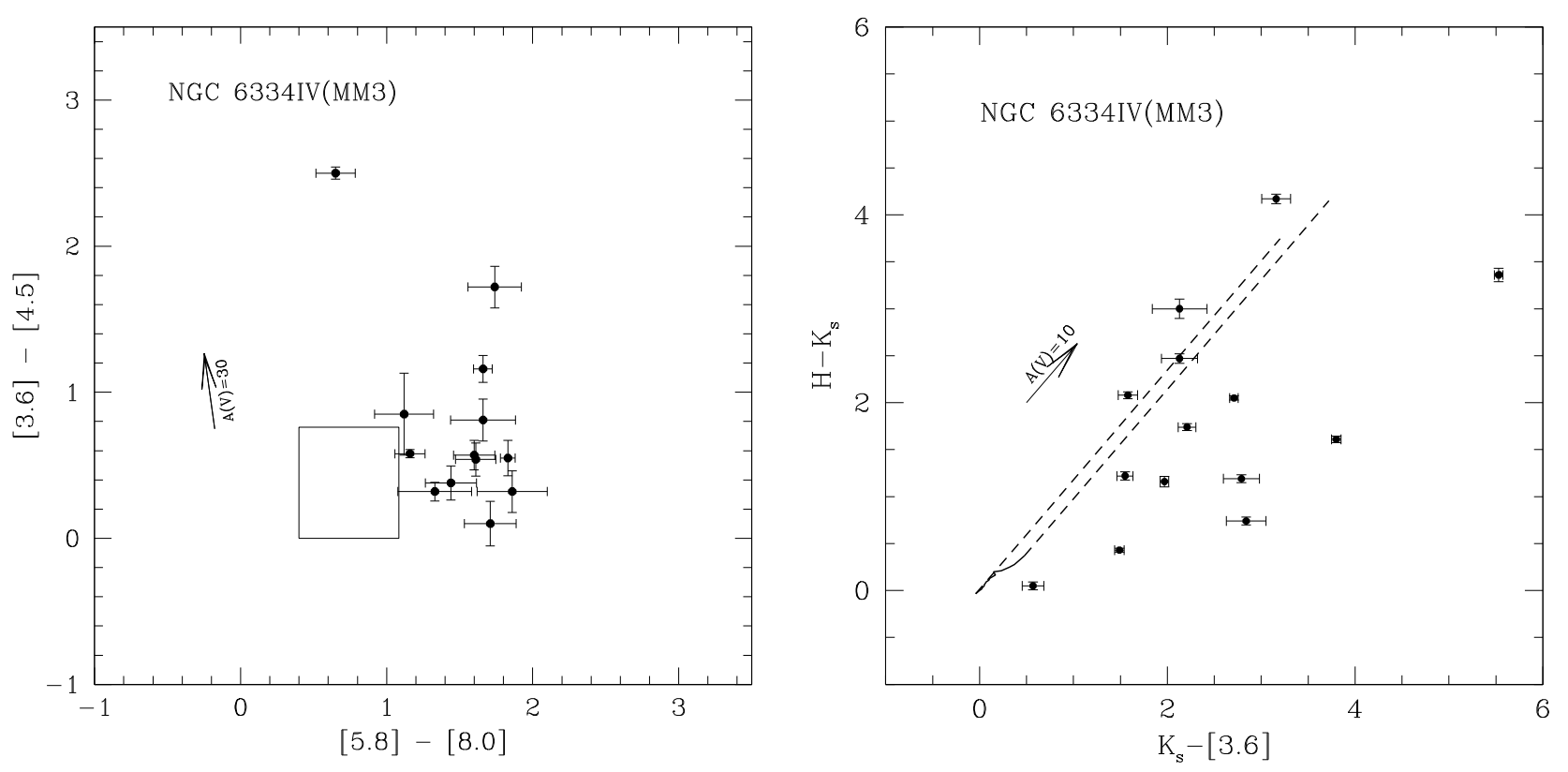

Fig. 6. Left panel: IRAC color-color diagram of the sources observed in an area of $75^{\prime \prime} \times 75^{\prime \prime}$ in NGC 6334 IV (MM3). The location of Class II YSOs (represented by a box) is taken from Allen et al. (2004). The reddening vector is also shown for $A_{V}=30$. Right panel: $H-K_{\mathrm{s}}$ versus $K_{\mathrm{s}}-$ [3.6] diagram. The solid line marks the locus of the main-sequence stars from Bessel \& Brett (1988), while the dashed lines define the reddening band from Rieke \& Lebofsky (1985).

Table 3. Coordinates, $H, K_{\mathrm{s}}$ and IRAC/Spitzer magnitudes of the sources with infrared excess.

\begin{tabular}{|c|c|c|c|c|c|c|c|c|c|}
\hline IRS & $\alpha \alpha(2000)$ & $\overline{\delta(2000)}$ & $H$ & $K_{\mathrm{s}}$ & {$[3.6]$} & [4.5] & [5.8] & {$[8.0]$} & $\overline{\mathrm{IID}}$ \\
\hline & $\mathrm{h} \mathrm{m} \quad \mathrm{s}$ & $\circ, \quad \prime$ & mag & mag & mag & mag & mag & mag & \\
\hline 9 & 172020.26 & -355503.9 & $13.52(0.01)$ & $11.25(0.01)$ & $8.76(0.04)$ & $8.44(0.05)$ & $6.88(0.14)$ & $5.55(0.21)$ & HHS9 \\
\hline 10 & 172020.69 & -355538.7 & $14.11(.0 .02)$ & $12.27(0.01)$ & $8.70(0.04)$ & $8.40(0.04)$ & $5.51(0.04)$ & & HHS 12 \\
\hline 11 & 172020.71 & -355505.1 & $10.95(0.04)$ & $10.07(0.01)$ & $7.82(0.02)$ & 7.24(0.02) & $6.12(0.05)$ & $4.96(0.09)$ & HHS 10 \\
\hline 12 & 172020.74 & -355515.9 & $11.29(0.03)$ & $10.83(0.01)$ & $10.67(0.11)$ & $10.35(0.09)$ & 7.96(0.18) & $6.10(0.16)$ & HHS 11 \\
\hline 13 & 172020.89 & -355459.1 & & $15.56(0.05)$ & $10.49(0.07)$ & $9.95(0.09)$ & 7.62(0.07) & $6.01(0.12)$ & \\
\hline 14 & 172021.18 & -355534.1 & $14.63(0.04)$ & $13.50(0.01)$ & $11.05(0.21)$ & & & & HHS 13 \\
\hline 15 & 172021.25 & -355437.6 & $15.14(0.03)$ & $14.65(0.02)$ & $11.19(0.09)$ & $10.64(0.08)$ & 7.71(0.04) & $5.88(0.03)$ & HHS49 \\
\hline 16 & 172021.63 & -355541.5 & $16.03(0.04)$ & $14.63(0.02)$ & $12.05(0.19)$ & $11.92(0.22)$ & & 7.94(0.25) & MM4? \\
\hline 17 & 172021.99 & -355456.3 & $12.20(0.02)$ & $10.28(0.05)$ & $11.51(0.01)$ & $9.01(0.04)$ & $7.51(0.06)$ & $6.86(0.12)$ & HHS16 \\
\hline 18 & 172022.91 & -355526.3 & & $15.77(0.04)$ & $10.00(0.08)$ & $9.19(0.12)$ & $6.89(0.13)$ & $5.23(0.18)$ & G351.24+0.65, neb. \\
\hline 19 & 172023.19 & -355520.6 & & $15.66(0.04)$ & $10.01(0.06)$ & $9.63(0.10)$ & $6.73(0.09)$ & $5.29(0.15)$ & MIRS2 \\
\hline 20 & 172023.52 & -355541.7 & & $16.51(0.05)$ & $11.37(0.23)$ & $10.52(0.16)$ & $6.91(0.09)$ & $5.79(0.18)$ & neb. \\
\hline 21 & 172023.61 & -355503.9 & $17.65(0.06)$ & $14.38(0.02)$ & $8.76(0.04)$ & $6.86(0.02)$ & & & IRS7 \\
\hline 22 & 172024.19 & -355518.7 & & $16.26(0.06)$ & $9.49(0.08)$ & $9.39(0.13)$ & $6.26(0.13)$ & $4.55(0.12$ & neb. \\
\hline 23 & 172024.58 & -355527.9 & $12.46(0.03)$ & $11.24(0.01)$ & $9.69(0.08)$ & $9.12(0.06)$ & $6.47(0.10)$ & $4.87(0.10)$ & \\
\hline 24 & 172024.87 & -355533.5 & & & $10.79(0.13)$ & $9.07(0.06)$ & $7.26(0.13)$ & $5.52(0.13)$ & neb. \\
\hline 25 & 172025.77 & -355450.1 & & $15.19(0.03)$ & $10.96(0.07)$ & $9.80(0.06)$ & $6.93(0.04)$ & $5.27(0.05)$ & \\
\hline
\end{tabular}

\subsection{IR-MM3}

As seen in Fig. 1, IR-MM3 (IRS 8E) is a very red object located at the apex of the polarized infrared reflection nebula IRN IV-3 (Hashimoto et al. 2008). The source coincides with the OH maser found by Brooks \& Whiteoak (2001) and is within the positional error of the continuum millimeter source MM3. Using the mid-infrared flux densities of Table 2, the $K_{\mathrm{s}}$ photometry of Table 1 and the sub-millimeter and millimeter fluxes reported by Sandell (1999), we derived the spectral energy distribution (SED) of IR-MM3 shown in Fig. 7. The spectral points have been compared with the spherically symmetric dust envelope model DUSTY (Ivezić \& Elitzur 1997) in order to derive some plausible physical parameters. We assumed standard optical properties for the dust grains (Draine \& Lee 1984), temperature of the central star of $15000 \mathrm{~K}$ and of the inner radius $T_{i}=1300 \mathrm{~K}$. The best fit to the data (solid line in Fig. 7) was obtained for a dust density distribution of the type $\eta(r) \propto r^{-0.3}$ and for the parameters reported in Table 4, where $r_{\mathrm{i}}$ and $r_{\mathrm{o}}$ are the inner and outer radii of the circumstellar envelope, and $T_{\mathrm{d}}$ is the temperature of the dust at $r_{\mathrm{o}} . A_{V}$ is the total extinction in the $V$ band. The bolometric luminosity, $L_{\text {bol }}$, was derived from the observed total flux, $F_{\mathrm{T}}$, assuming a distance for the region of $1.61 \mathrm{kpc}$ (Persi \& Tapia 2008). In addition, we derived the infrared spectral index $\alpha_{\mathrm{IR}}=\mathrm{d} \log \lambda F(\lambda) / \mathrm{d} \log \lambda$ computed 


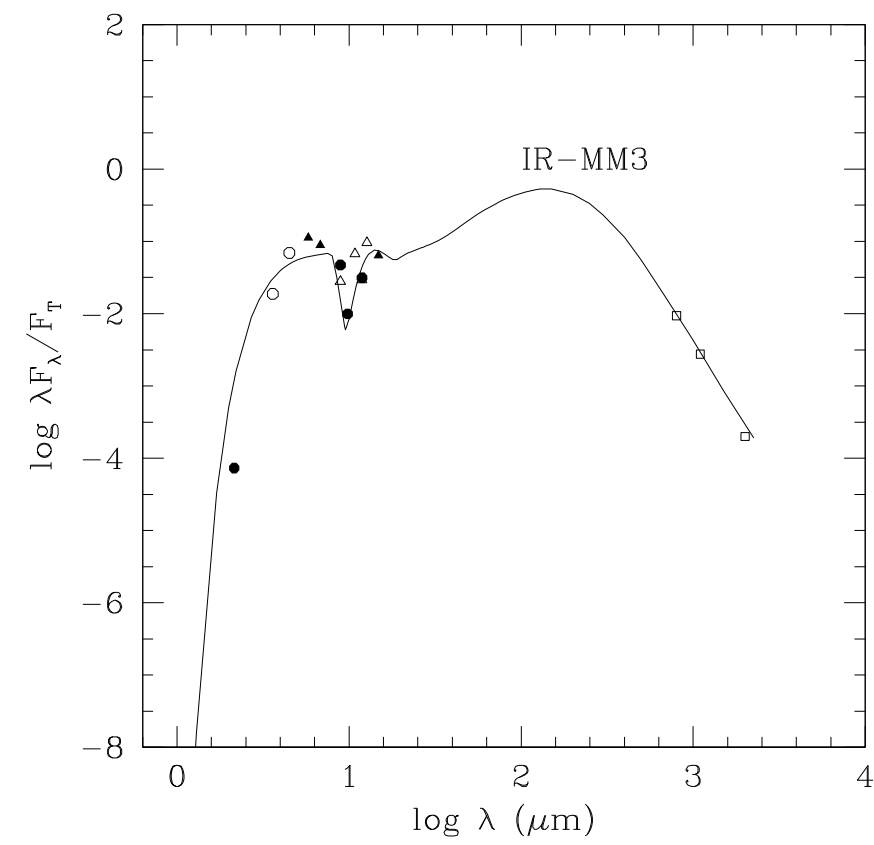

Fig. 7. SED of IR-MM3. The solid line is the spherically symmetric dust model obtained from the DUSTY code with the parameter reported in Table 4. The different symbols are: filled and open circles from ESO-TIMMI2 and IRAC observations, filled and open triangles from ISOCAM and CID photometry. The sub(millimeter) and millimeter observations (open squares) are from Sandell (1999).

from 2.15 to $11.9 \mu \mathrm{m}$. The values of $\alpha_{\mathrm{IR}}=3.5$ and $L_{\mathrm{bol}}=985 L_{\odot}$ confirm that IR-MM3 is a very young star, with a luminosity lower than that of a B3 ZAMS.

\section{3. $G 351.24+0.65$}

G351.24+0.65 is a faint radio source discovered by Moran et al. (1990) at $6 \mathrm{~cm}$. The source was also detected at $3.6 \mathrm{~cm}$ by De Pree et al. (1995). Comparing the two observed flux densities, these authors suggest that G351.24+0.65 is an optically thin HII region ionized by a B0 ZAMS star. From a new analysis of De Pree et al.'s observations, Rodríguez (private communication) found that this radio source is double with a separation between the two peaks of around $8.8^{\prime \prime}$ in the east-west direction, as shown in Fig. 8. This HII region is located between the millimeter sources MM3 and MM4. In Fig. 8, the $3.6 \mathrm{~cm}$ contours are reported superimposed on our $K_{\mathrm{s}}$ image. Sources IRS 18 and IRS 19 (MIRS2), with large infrared excess are within the boundaries of the radio HII region. In particular, source IRS 18, embedded in a nebulosity, coincides with the western peak of G351.24+0.65 and shows a steep SED (Fig. 9). IRS 19 (MIRS2), detected also at $11.9 \mu \mathrm{m}$, has an infrared spectral index $\alpha_{\mathrm{IR}}=1.92$ computed between 2.15 and $11.9 \mu \mathrm{m}$.. Both these young stellar objects may contribute to the ionization of the HII region.

The presence of patchy dust structures is inferred from the knotty $\mathrm{Br} \gamma$ emission which clearly departs from the morphology seen in the radio-continuum. Four bright $\mathrm{Br} \gamma$ knots have been identified inside the radio HII region. Their positions are listed in Table 5 and indicated in Fig. 1. The distribution of the dust forming a circumstellar disk would help towards explaining the elongated molecular hydrogen knots A3, A4 and A5 (see next subsection). It is noteworthy that knot B3, coinciding with
Table 4. Model parameters of IR-MM3 from the DUSTY code.

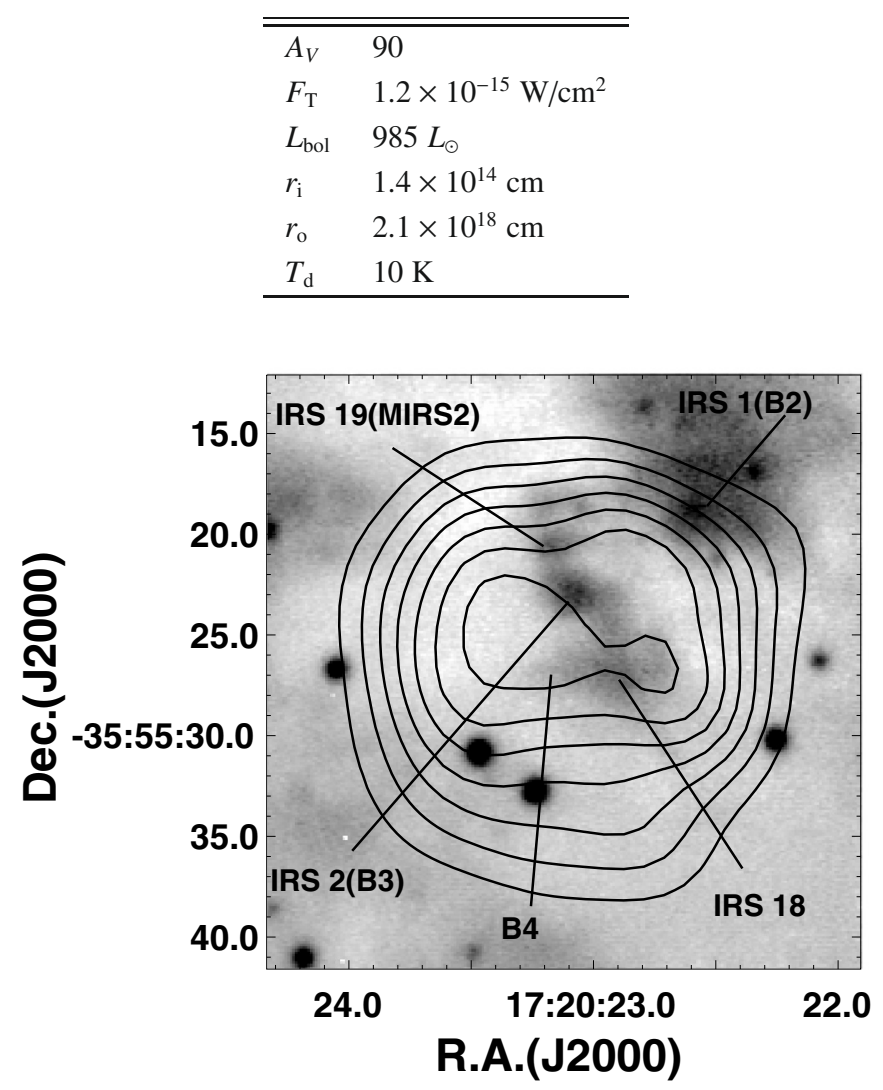

Fig. 8. $K_{\mathrm{S}}$ image of the area of the radio HII region G351.24+0.65 with the VLA $3.6 \mathrm{~cm}$ contours overlaid (Rodriguez, private communication). The bright $\mathrm{Br} \gamma$ knots and the infrared-excess sources are labeled.

the red IRS 2, is close to the position of another of the radiocontinuum maxima (see Fig. 8).

\subsection{Hydrogen molecular emission}

Strong $\mathrm{H}_{2} v=1 \rightarrow 0 \mathrm{~S}(1)$ line emission, most probably caused by shocks, has been detected in six positions of NGC 6334 IV (MM3) as shown by the color-coded image presented in Fig. 1. The coordinates, $\mathrm{H}_{2}$ line fluxes, and the measured sizes from our calibrated continuum-subtracted image are given in Table 6 . We conservately estimate an accuracy of $\sim 30 \%$ in deriving the $\mathrm{H}_{2}$ line fluxes. The $\mathrm{H}_{2}$ knots $\mathrm{A} 1$ and $\mathrm{A} 2$ are very close to the millimeter peak MM3, while A3, A4 and A5 are located along the line joining MM4 and MM3 and through the HII region G351.24+0.65, including the red objects IRS 18 and IRS 19 (see Fig. 9). This morphology suggests the presence of an outflow in this direction. With the available information, nevertheless, it is not possible to determine which of these, or another source, is the engine of such outflow. On the other hand, the north-eastern $\mathrm{H}_{2}$ knots $\mathrm{A} 1$ and $\mathrm{A} 2$ may originate in a different system, probably a less energetic one, as no ionized gas has been detected in the radio-continuum in its vicinity. Alternatively, these shocked knots may be formed at the end of a bipolar cavity formed by the same event originating in G351.24+0.65, a scenario supported by the arc-like appearance of A2 (Fig. 1). High resolution $\mathrm{CO}$ observations are needed to confirm the presence of a molecular outflow. Finally, the $\mathrm{H}_{2}$ knot A6 is at the apex of 


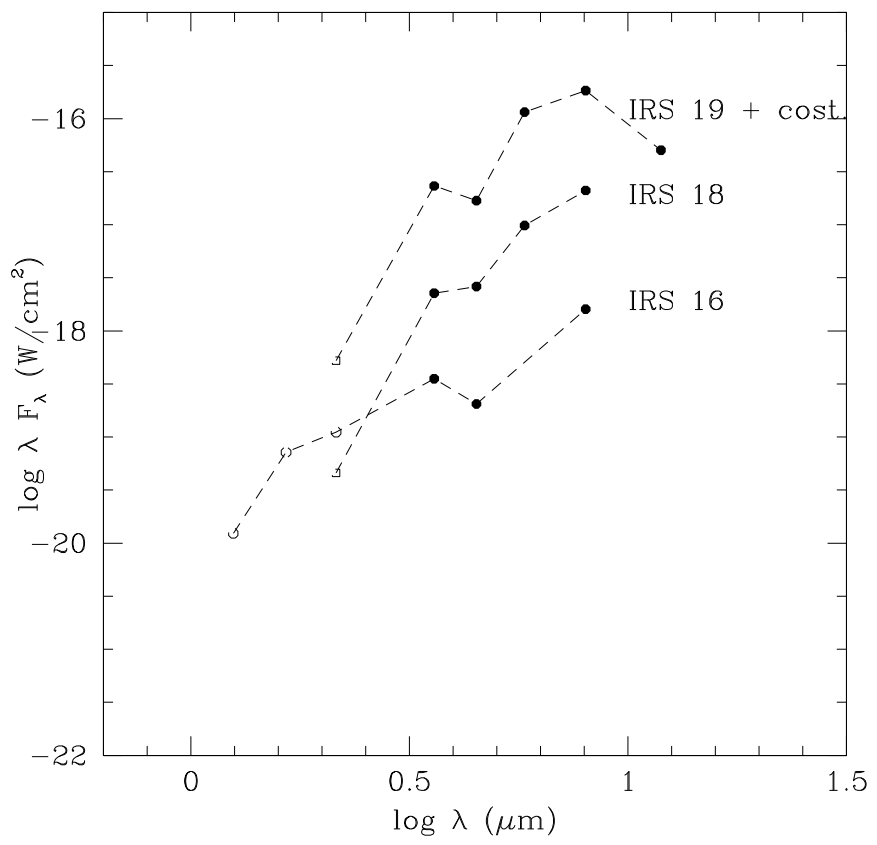

Fig. 9. Spectral energy distributions (SEDs) of IRS 16, IRS 18 and IRS 19.

Table 5. Coordinates of the Br $\gamma$ knots.

\begin{tabular}{|c|c|c|c|}
\hline$\overline{\mathrm{Br} \gamma \text { knots }}$ & $\begin{array}{l}\alpha(2000) \\
\mathrm{h} \mathrm{m} \mathrm{s}\end{array}$ & $\begin{array}{l}\delta(2000) \\
\circ \quad, \quad \prime\end{array}$ & Id. \\
\hline B1 & 172023.18 & -355520.6 & \\
\hline B2 & 172022.65 & -355519.1 & IRS 1 \\
\hline B3 & 172023.07 & -355523.0 & IRS 2 \\
\hline B4 & 172022.91 & -355526.8 . & \\
\hline
\end{tabular}

the infrared reflection nebula IRN IV-6, and was labeled IRS 3 by Persi et al. (2000).

\section{Conclusions}

We have obtained high sensitivity and sub-arcsec resolution near and mid infrared images of the eastern part of the star forming region NGC 6334 IV. From the analysis of these images and from the IRAC/Spitzer and ISOCAM images we derived the following conclusions:

1) In an area of approximately $75^{\prime \prime} \times 75^{\prime \prime}$ containing the millimeter sources MM3, MM4 and the HII region G351.24+0.65, we have found 18 point-like sources with significant excess emission at $\lambda \geq 2 \mu \mathrm{m}$ indicating the presence of a young stellar population in this region (see Table 3 and Fig. 5).

2) One of these sources, IR-MM3(IRS8E), coinciding with the $\mathrm{OH}$ maser source, and located within the positional uncertainty of the millimeter continuum source MM3, has a very steep infrared energy distribution (Fig. 7 right panel) with the infrared spectral index $\alpha(\mathrm{IR})=3.5$ and a bolometric luminosity of $985 L_{\odot}$. These parameters indicate that IR-MM3 is a very young star later than a B3 ZAMS.

3) Three other objects (IRS 16, IRS 18 and IRS 19 in Table 4) show a steep infrared energy distribution (Fig. 9). IRS 18
Table 6. Coordinates and line fluxes of the $\mathrm{H}_{2}$ knots.

\begin{tabular}{|c|c|c|c|c|}
\hline \multirow[t]{2}{*}{$\overline{\mathrm{H}} \mathrm{H}_{2}$ knots } & $\overline{\alpha \alpha(2000)}$ & $\overline{c / \delta(2000)}$ & \multirow{2}{*}{$\begin{array}{l}\mathrm{H}_{2} v=1 \rightarrow 0 \mathrm{~S}(1) \\
\mathrm{erg} \mathrm{s}^{-1} \mathrm{~cm}^{-2}\end{array}$} & \multirow{2}{*}{$\begin{array}{l}\text { Size } \\
,\end{array}$} \\
\hline & $\mathrm{h} \mathrm{m} \quad \mathrm{s}$ & $\circ, \quad \prime$ & & \\
\hline A1 & 172024.55 & -355501.9 & $5.7 \times 10^{-15}$ & 0.8 \\
\hline $\mathrm{A} 2$ & 172024.78 & -355503.3 & $3.0 \times 10^{-15}$ & 2.0 \\
\hline A3 & 172022.70 & -355530.9 & $4.4 \times 10^{-15}$ & 1.7 \\
\hline A4 & 172022.48 & -355534.2 & $0.9 \times 10^{-15}$ & 1.3 \\
\hline A5 & 172022.94 & -355524.3 & $1.1 \times 10^{-15}$ & 1.3 \\
\hline A6 & 172023.25 & -355506.8 & $1.9 \times 10^{-15}$ & 2.6 \\
\hline
\end{tabular}

and IRS 19 are probably the ionizing stars of the optically thin HII region G351.24+0.65, while IRS 16 probably is connected to the MM4 millimeter source.

4) Six hydrogen molecular emission knots have been detected in the region (Fig. 1). The young source IR-MM3 is the exciting source of the knots A1 and A2, while the knots A3, A4 and A5 aligned along the direction of the MM3 and MM4 millimeter peaks suggest the presence of a molecular outflow.

5) The extended nebulosities seen at 5.8 and $8.0 \mu \mathrm{m}$ with IRAC (Fig. 4, left panel) and at 5.8 and $6.8 \mu \mathrm{m}$ with ISOCAM (Fig. 4, right panel) indicate the presence of extended PAH emission in this area.

In summary, we can state that NGC 6334 IV(MM3) is a very active center of star formation inside the molecular complex.

Acknowledgements. M.T. acknowledges PAPIIT/DGAPA grant No. IN102607. We thank L. F. Rodríguez for sending his reanalyzed VLA $3.6 \mathrm{~cm}$ archived image of G351.24+0.65. This paper is partially based on observations made with the Spitzer Space Telescope, which is operated by the Jet Propulsion laboratory, California Institute of Techonology (CIT) under NASA contract 1407, and with the ISO satellite (ISOCAM instrument). ISO is an ESA project funded by ESA member states.

\section{References}

Allen, L. E, Calvet, N., D'Alessio, P., et al. 2004, ApJS, 154, 363 Benjamin, R. A., Churchwell, E., Babler, B. L., et al. 2003, PASP, 115, 953 Bessel, M. S., \& Brett, J. M. 1988, PASP, 100, 1134 Brooks, K. J., \& Whiteoak, J. B. 2001, MNRAS, 320, 465

Cesarsky, C. J., Abergel, A., Agnése, P., et al. 1996, A\&A, 315, L32 De Pree, C. G., Rodríguez, L. F., Dickel, H. R., \& Goss, W. M. 1995, ApJ, 447, 220

Draine, B. T., \& Lee, H. M. 1984 ApJ, 284, 89

Fazio, G. G., Hora, J. L., Allen, L. E., et al. 2004, ApJS, 154, 10 Harvey, P. M., Hyland, A. R., \& Straw, S. M. 1987, ApJ, 317, 173

Hashimoto, J., Tamura, M., Kandori, R., et al. 2008, ApJ, 677, L39 Ivezić, Z., \& Elitzur, M. 1997, MNRAS, 287, 799

Jones, T. J., Woodward, M. L., Boyer, R. D., et al. 2005, ApJ, 620, 731

Kessler, M. F., Steinz, J. A., Anderegg, M. E., et al. 1996, A\&A, 315, L27 Kraemer, K. E., Deutsch, L. K., Jackson, J. M., et al. 1999, ApJ, 516, 817 Moran, J. M., Rodríguez, L. F., \& Backer, D. C. 1990, ApJ, 348, 147 Persi, P., \& Tapia, M. 2008, in Handbook Star Forming Regions Vol. II The Southern Sky, ASP Conf. Ser., ed. B. Reipurth, in press

Persi, P., Tapia, M., \& Roth, M. 2000, A\&A, 357, 1020

Persi, P., Tapia, M., \& Smith, H. A. 2006, A\&A, 445, 971

Persson, S. E., Murphy, D. C., Krzeminski, W., et al. 1998, AJ, 116, 2475

Rieke, G. K., \& Lebofsky, M. J. 1985, ApJ, 288, 618

Rodríguez, L. F., Cantó, J., \& Moran, J. M. 1982, ApJ, 225, 103

Rodríguez, L. F., Cantó, J., \& Moran, J. M. 1988, ApJ, 333, 801

Salas, L., Cruz-González, I., \& Tapia, M. 2006, Rev. Mex., 42, 273

Sandell, G. 1999, A\&A, 343, 281

Stetson, P. B. 1987, PASP, 99, 191

Werner, M. W., Roelling, T. L., Low, F. J., et al. 2004, ApJS, 154, 1 